\title{
Electronic Instability at High Flux-Flow Velocities in High-Tc Superconducting Films
}

Doettinger, S. G.; Huebener, R. P.; Gerdemann, R.; Kyhle, Anders; Anders, S.; Träuble, T. G.; Villégier, J. C.

Published in:

Physical Review Letters

Link to article, DOI:

10.1103/PhysRevLett.73.1691

Publication date:

1994

Document Version

Publisher's PDF, also known as Version of record

Link back to DTU Orbit

Citation $(A P A)$ :

Doettinger, S. G., Huebener, R. P., Gerdemann, R., Kyhle, A., Anders, S., Träuble, T. G., \& Villégier, J. C. (1994). Electronic Instability at High Flux-Flow Velocities in High-Tc Superconducting Films. Physical Review Letters, 73(12), 1691-1694. https://doi.org/10.1103/PhysRevLett.73.1691

\section{General rights}

Copyright and moral rights for the publications made accessible in the public portal are retained by the authors and/or other copyright owners and it is a condition of accessing publications that users recognise and abide by the legal requirements associated with these rights.

- Users may download and print one copy of any publication from the public portal for the purpose of private study or research.

- You may not further distribute the material or use it for any profit-making activity or commercial gain

- You may freely distribute the URL identifying the publication in the public portal 


\title{
Electronic Instability at High Flux-Flow Velocities in High- $T_{c}$ Superconducting Films
}

\author{
S. G. Doettinger, ${ }^{1}$ R. P. Huebener, ${ }^{1}$ R. Gerdemann, ${ }^{1}$ A. Kühle, ${ }^{2}$ S. Anders, ${ }^{1}$ T. G. Träuble, ${ }^{1}$ and J. C. Villégier ${ }^{3}$ \\ ${ }^{1}$ Physikalisches Institut, Experimentalphysik II, Universität Tübingen, Morgenstelle 14, D-72076 Tübingen, Germany \\ ${ }^{2}$ Physics Department, Technical University of Denmark, DK-2800 Lyngby, Denmark \\ ${ }^{3}$ Laboratoire d'Electronique et de Technologie de l'Informatique-Commissariat à l'Energie Atomique-Technologies Avancées, \\ 85x, F-38041 Grenoble, France
}

(Received 18 April 1994)

\begin{abstract}
At high flux-flow velocities in type-II superconductors the nonequilibrium distribution of the quasiparticles leads to an electronic instability and an abrupt switching into a state with higher electric resistivity, as predicted by Larkin and Ovchinnikov (LO). We report the first observation of this effect in a high-temperature superconductor, namely in epitaxial $c$-axis oriented films of $\mathrm{YBa}_{2} \mathrm{Cu}_{3} \mathrm{O}_{7-\delta}$. Using the LO theory, we have extracted from our results the inelastic quasiparticle scattering rate $\tau_{\text {in }}^{-1}$, which strongly decreases with decreasing temperature below $T_{c}$.
\end{abstract}

PACS numbers: 74.25.Fy, 74.60.Ge

Vortex motion and energy dissipation in the mixed state of high- $T_{c}$ superconducting films has been intensively studied during recent years. These studies focused predominantly on the mechanisms controlling vortex motion in the limit of low values of the driving Lorentz force or thermal force. Hence, in the past the onset of dissipation and the vortex dynamics in the limit of low vortex velocities occupied the center of interest. In the present paper, we report for the first time on experiments dealing with the opposite limit, namely the maximum possible vortex velocity established at high values of the driving force. In this regime an interesting electronic instability has been predicted about 20 years ago by Larkin and Ovchinnikov (LO) [1], resulting in a discontinuity in the current-voltage characteristic (IV) and an abrupt switching of the sample into another state of higher electric resistivity. The experimental observation of this effect for low- $T_{c}$ superconductors has been reported some years ago [2,3], and reasonable agreement with the LO theory has been found. In this phenomenon, the relaxation rate of the quasiparticle energy plays a central role, and it is this feature which makes an experimental study of this effect in the high-temperature superconductors particularly interesting. Recently, inelastic scattering between quasiparticles has played an increasing role in the theoretical discussions of the electronic structure and the mechanism underlying high-temperature superconductivity [4,5]. Furthermore, inelastic quasiparticle scattering and its temperature dependence has become an important issue in the interpretation of recent experiments on the high-frequency conductivity $[6,7]$, infrared optics [8], and thermal conductivity [9] of hightemperature superconductors.

We report on the first experimental observation in a high-temperature superconductor of the electronic instability due to quasiparticle excitation in the normal vortex core at high flux-flow velocities, as predicted by LO [1]. The effect manifests itself in an abrupt increase of the electric resistance of the sample. Our measure- ments were performed with epitaxial $c$-axis oriented $\mathrm{YBa}_{2} \mathrm{Cu}_{3} \mathrm{O}_{7-\delta}$ films and clearly indicate that the inelastic electron-electron scattering rate strongly decreases with decreasing temperature below $T_{c}$. This temperature dependent quasiparticle scattering plays an increasing role in recent discussions of the mechanism for high-temperature superconductivity and of the underlying electronic structure.

The main point of the LO theory is the following. In the presence of the electric field resulting from currentinduced vortex motion the nonequilibrium distribution of the quasiparticles in the superconductor is shifted upwards in energy compared to the equilibrium distribution. As a consequence, quasiparticles leave the potential well of the vortex core, the vortex core shrinks, viscous damping of the vortex motion is reduced, and the vortex velocity normalized to the driving force becomes larger. According to LO, the viscous damping coefficient $\eta$ at vortex velocity $v_{\varphi}$ is

$$
\eta\left(v_{\varphi}\right)=\frac{\eta(0)}{1+\left(v_{\varphi} / v_{\varphi}^{*}\right)^{2}},
$$

where $\eta(0)$ is the damping coefficient at $v_{\varphi}=0$ and $v_{\varphi}^{*}$ a critical vortex velocity at which the damping force $\eta v_{\varphi}$ attains a maximum. Because of this effect the $I V$ characteristic is curved upwards and at the velocity $v_{\varphi}^{*}$ an instability point is reached where the sample switches into another state. This instability point manifests itself as a distinct discontinuity in the $I V$ characteristic. The nonequilibrium quasiparticle distribution and, hence, the value of $v_{\varphi}^{*}$ depends sensitively on the inelastic electronelectron scattering rate $\tau_{\text {in }}^{-1}$ which tends to move the quasiparticle system back to its equilibrium distribution. A qualitative discussion of this point based on simple physical concepts has been given elsewhere [3]. For the critical velocity $v_{\varphi}^{*}$ LO obtain the expression

$$
v_{\varphi}^{*}=\frac{D[14 \zeta(3)]^{1 / 2}\left(1-T / T_{c}\right)^{1 / 2}}{\pi \tau_{\text {in }}} .
$$


Here $D$ is the quasiparticle diffusion coefficient $(D=$ $v_{F} l / 3$, with $v_{F}$ the Fermi velocity and $l$ the electron mean free path); $\zeta(x)$ is the Riemann zeta function, and $\tau_{\text {in }}$ is the inelastic quasiparticle scattering time. The flux-flow voltage corresponding to $v_{\varphi}^{*}$ is

$$
\underline{\mathbf{v}}^{*}=-\left(\underline{\boldsymbol{v}}_{\varphi}^{*} \times \underline{\mathbf{B}}\right) L,
$$

where $\underline{\mathbf{B}}$ is the magnetic field and $L$ the sample length between the voltage contacts. As discussed by LO, at voltages $V \leq V^{*}$ the curved $I V$ characteristic is expected to follow the equation

$$
I-I_{c}=\left[\frac{V}{1+\left(V / V^{*}\right)^{2}}+V\left(1-\frac{T}{T_{c}}\right)^{1 / 2}\right] \frac{1}{R_{f}} .
$$

where $I_{c}$ and $R_{f}$ are the critical current and the flux-flow resistance, respectively. In an experimental study of this electronic nonequilibrium phenomenon great care must be exercised for eliminating heating effects.

Our sample films had a thickness of $60-100 \mathrm{~nm}$ and were deposited on single-crystalline $\mathrm{MgO}$ substrates by laser ablation. Microfabrication of the four-point sample geometry was performed by standard photolithography. For attaching the current and voltage leads, silver contact pads as large as $2 \times 2 \mathrm{~mm}^{2}$ area were placed on top of the superconducting film in this way minimizing sample heating due to the contact resistance. The length (between voltage leads) and width of the sample film was typically 100 and $20 \mu \mathrm{m}$, respectively. The $\mathrm{MgO}$ substrate was in good thermal contact with a temperature-regulated copper block of the sample holder, allowing the variation of the sample temperature between 10 and $300 \mathrm{~K}$. The samples studied in this paper had a sharp resistive transition within typically $2 \mathrm{~K}$ and zero resistance critical temperature $T_{c 0}$ of $89 \mathrm{~K}$. At $100 \mathrm{~K}$ the resistivity was $70-80 \mu \Omega \mathrm{cm}$. A magnetic field could be applied parallel to the $c$ axis.

For substrates we have selected $\mathrm{MgO}$, since the thermal conductivity in this material is about 10 times higher compared to single-crystalline $\mathrm{SrTiO}_{3}[10]$. The heat transfer coefficient between the $\mathrm{YBa}_{2} \mathrm{Cu}_{3} \mathrm{O}_{7-\delta}$ film and $\mathrm{MgO}$ is about $2000 \mathrm{~W} / \mathrm{cm}^{2} \mathrm{~K}$ [11], thereby limiting the temperature rise due to the power dissipation in the sample films measured by us to values not larger than about $0.5 \mathrm{~K}$. In addition, in order to reduce effects due to substrate heating to an acceptable low level, we found that it was necessary to measure the $I V$ characteristic beyond the instability point using a rapid single-pulse method. The current was swept up and down in a single triangular pulse, and the voltage was recorded simultaneously. The time scale finally adopted for an upsweep was typically $20 \mathrm{~ms}$. Our instrumentation consisted of a HP 8165 Programmable Signal Source and a Tektronix model 11201A Digitizing Oscilloscope combined with a PAR model 113 preamplifier. We have studied three different epitaxial $\mathrm{YBa}_{2} \mathrm{Cu}_{3} \mathrm{O}_{7-\delta}$ films, all showing similar results.
A typical set of experimental results is shown in Fig. 1. Here the applied current $I$ and the voltage $V$ measured during a single pulse is plotted versus time. The maximum current was selected such that the discontinuity in the voltage could clearly be observed. At $V=225 \mathrm{mV}$ the temporal dependence of the voltage abruptly changes its slope turning into a nearly vertical branch. Note that the triangular current pulse slightly extends beyond this point. before the downsweep of the current begins. The voltage $V=225 \mathrm{mV}$ is interpreted as the flux-flow voltage $V^{*}$. corresponding to the critical flux-flow velocity $v_{\varphi}^{*}$ according to Eq. (3). We have also confirmed that the functional form of Eq. (4) is well satisfied by the experimentally observed $I V$ characteristics for $V \leq V^{*}$. The velocity $v_{\psi}^{*}$ is plotted as a function of magnetic field $B$ for three temperatures in Fig. 2. As expected from Eq. (2), we see that $v_{4}^{*}$ is nearly independent of $B$. This clearly allows an accurate determination of $v_{\varphi}^{*}$. In Fig. 3 we plot the velocity $v_{\varphi}^{*}$ versus temperature.

Our interpretation of the values of $V^{*}$ and $v_{\varphi}^{*}$ in terms of an electronic instability of the type discussed by LO (in contrast to an instability dominated by heating) is supported, among others, by the following experimental facts. (1) $v_{4}^{*}$ was found to be independent from $B$ as expected from Eq. (2). On the other hand, the voltage $V^{*}$ increased proportional to $B$, whereas the current $I^{*}$, at which $V^{*}$ is reached, remained approximately constant. Although in some cases by changing $B$ the power dissipation $I^{*} V^{*}$ varied by a factor as large as 10 , the independence of $v_{\varphi}^{*}$ from $B$ remained unaffected. As shown theoretically [12] and demonstrated experimentally [13] sample heating leads to distinct deviations from the proportionality between $V^{*}$ and $B$ and from the $B$ independence of $I^{*}$. Both kinds of deviations we did not observe. However, using $\mathrm{SrTiO}_{3}$ instead of $\mathrm{MgO}$ as substrate material, both kinds of deviations clearly appeared indicating effects due to substrate heating. (2) Variation of the time scale of the upsweep of the current from $20 \mathrm{~ms}$ upwards by a factor of 3 or downwards by

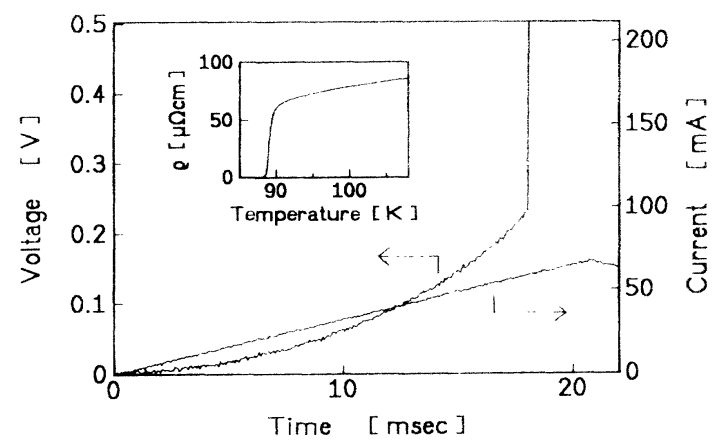

FIG. 1. Voltage (left vertical axis) and current (right vertical axis) versus time. $T=72 \mathrm{~K}, B=1.8 \mathrm{~T}$. The inset shows the resistivity versus temperature near the superconducting transition. 


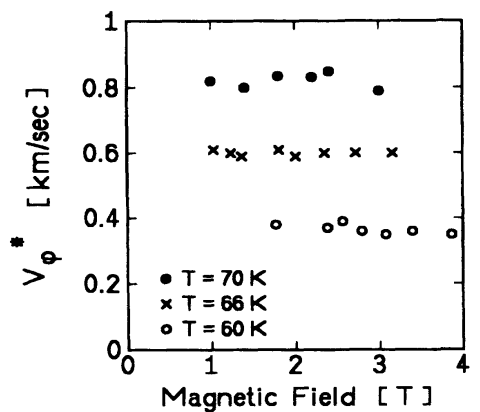

FIG. 2. Critical flux-flow velocity $v_{\varphi}^{*}$ versus magnetic field for three temperatures. Same sample as in Fig. 1.

a factor of 50, left the values of $V^{*}$ and $I^{*}$ unchanged within $1 \%$. However, by increasing this time scale beyond about $100 \mathrm{~ms}$, we noted the first tendency for an increase of $V^{*}$ and a decrease of $I^{*}$, as expected when sample heating starts to play a role. (3) By limiting the maximum current of the triangular current pulse to values $I \leq I^{*}$, at which the voltage instability is reached, the voltage could be reversibly traced backwards during the downturn of the current. However, if this maximum current exceeded $I^{*}$, the large increase in voltage and power dissipation caused thermal hysteresis and a deviation of this reversible behavior.

Because of the arguments given above, we feel confident that our interpretation of the observed break of the $I V$ characteristic at $V^{*}$ or $v_{\varphi}^{*}$ in terms of an electronic instability and not a thermal one is justified. Using Eq. (2) derived by $\mathrm{LO}$ for calculating the inelastic scattering rate $\tau_{\text {in }}^{-1}$, we obtain the plot of $\tau_{\text {in }}^{-1}$ versus temperature shown in Fig. 4. In this calculation we have used the values $v_{F}=1 \times 10^{7} \mathrm{~cm} / \mathrm{s} \mathrm{[14]} \mathrm{and} l=5 \mathrm{~nm} \mathrm{[15].}$

As seen in Fig. 4, again we clearly find the rapid decrease of $\tau_{\text {in }}^{-1}$ with decreasing temperature below $T_{c}$, as observed already in Refs. [6-9]. Our determination of $\tau_{\text {in }}^{-1}$ could not be extended appreciably above $82 \mathrm{~K}$, since at these temperatures the scattering rate $\tau_{\text {in }}^{-1}$ quickly approaches the value for electron-phonon scattering of typically $10^{12}-10^{13} \mathrm{~s}^{-1}[6,7,15]$, thereby leaving the validity range of the LO theory. Experimentally, above

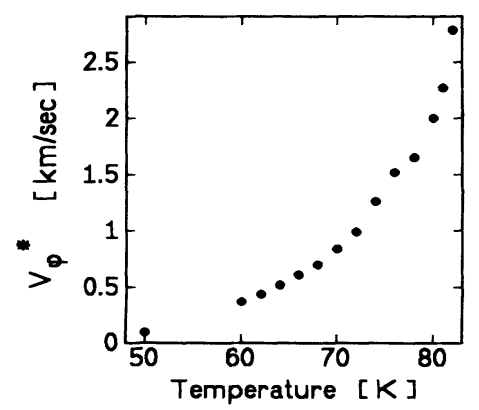

FIG. 3. Critical flux-flow velocity $v_{\varphi}^{*}$ versus temperature. Same sample as in Fig. 1.

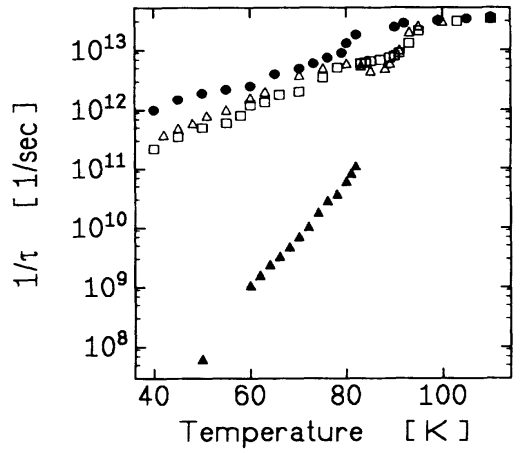

FIG. 4. Inelastic quasiparticle scattering rate $\tau_{\text {in }}^{-1}(\mathbf{\Lambda})$ obtained from Eq. (2) versus temperature. Same sample as in Fig. 1. For comparison we also show the scattering rate obtained from measurements of the microwave surface impedance and reported in Ref. [7] (O) and Ref. [6] (sample A $\square$, sample B $\triangle)$.

$82 \mathrm{~K}$ the value of $v_{\varphi}^{*}$ obtained from $V^{*}$ shows a distinct $B$ dependence, making an unambiguous determination of $v_{\varphi}^{*}$ impossible. For comparison we also show in Fig. 4 the quasiparticle scattering rate obtained recently from measurements of the microwave surface impedance of $\mathrm{YBaCuO}$ films [7] and single crystals [6]. It is interesting that the decrease of the scattering rate $\tau_{\text {in }}^{-1}$ with decreasing temperature displayed by our data is much more rapid than that of the microwave data. This difference in behavior can possibly be explained by the fact that the microwave results are much more sensitive to extrinsic scattering mechanisms (defects, impurities, surface irregularities, etc.) than the nonlinear $I V$ characteristic measured in our experiments. We emphasize that the $I V$ characteristic is only determined by the flux-flow process. Furthermore, it is only the inelastic quasiparticle scattering affecting the critical velocity $v_{\varphi}^{*}$, whereas the microwave losses are also due to the elastic processes. Although a detailed theoretical treatment still needs to be done, it appears that the scattering rate $\tau_{\text {in }}^{-1}$ is dominated by inelastic electron-electron scattering and that the opening of a gap in the quasiparticle energy spectrum below $T_{c}$ will be crucial for explaining the rapid suppression of $\tau_{\text {in }}^{-1}[16,17]$. A crude estimate of the temperature dependence of the scattering rate $\tau_{\text {in }}^{-1}$ can be obtained as follows. Noting that the Cooper pair binding energy is twice the energy gap $\Delta$, the thermally excited quasiparticle density $n_{\mathrm{qp}}$ is proportional to $\exp \left(-2 \Delta / k_{B} T\right)$. Hence, we find the proportionality $\tau_{\mathrm{in}}^{-1} \sim n_{\mathrm{qp}}^{2} \sim \exp \left(-4 \Delta / k_{B} T\right)$. Using for approximation the zero-temperature limit $\Delta(0) \approx 2 k_{B} T_{c}$, we obtain $\tau_{\text {in }}^{-1} \sim \exp \left(-8 T_{c} / T\right)$ and, finally, in our case of $T_{c}=89 \mathrm{~K} \tau_{\text {in }}^{-1} \sim \exp [(-710 \mathrm{~K}) / T]$. Fitting our data in Fig. 4 with the proportionality $\tau_{\text {in }}^{-1} \sim \exp \left(-T^{*} / T\right)$ we obtain $T^{*}=950 \mathrm{~K}$, which is reasonably close to our estimated value of $710 \mathrm{~K}$. On the other hand, electronphonon scattering is clearly inconsistent with this rapid 
suppression of $\tau_{\text {in }}^{-1}$ below $T_{c}$. Attempting to fit our data with a power law of the type $\tau_{\text {in }}^{-1} \sim T^{n}$, we obtained an unphysically high value $n \approx 14$.

The distance $v_{\varphi}^{*} \tau_{\text {in }}$ calculated from our data presented in Figs. 3 and 4 increases with decreasing temperature, with a value of $30 \mathrm{~nm}$ at $80 \mathrm{~K}$ and $1.5 \mu \mathrm{m}$ at $50 \mathrm{~K}$. This range of values is clearly much larger than the diameter of the normal vortex core (given by twice the coherence length). Noting that at a magnetic field of $1 \mathrm{~T}$ the intervortex distance is about $20 \mathrm{~nm}$, we see that, in the range of magnetic fields and temperatures we have studied, near the LO instability the nonequilibrium distribution of the quasiparticles extends over the whole volume of the superconductor.

To conclude, we have detected an interesting electronic instability in high- $T_{c}$ superconducting films due to the nonequilibrium quasiparticle distribution at high flux-flow velocities. Nonequilibrium effects in low- $T_{c}$ superconductors have been studied extensively some time ago, and summaries can be found in Refs. [18] and [19]. In high- $T_{c}$ superconductors this subject is just beginning to be investigated. Here the possible influence of the doping level on the nonequilibrium effects appears particularly interesting.

The authors acknowledge clarifying discussions with R. Gross and G. Schön and technical assistance from B. Mayer and A. Kittel.

[1] A. I. Larkin and Yu. N. Ovchinnikov, Zh. Eksp. Teor. Fiz. 68, 1915 (1975) [Sov. Phys. JETP 41, 960 (1976)].
[2] L. E. Musienko, I. M. Dmitrenko, and V. G. Volotskaya. Pis'ma Zh. Eksp. Teor. Fiz. 31, 603 (1980) |JETP Lett. 31, 567 (1980)].

131 W. Klein, R. P. Huebener, S. Gauss, and J. Parisi. J. Low Temp. Phys. 61, 413 (1985).

[4] W. E. Pickett, H. Krakauer, R. E. Cohen, and D. J. Singh. Science 255, 46 (1992).

[5] D. M. Newns. H. R. Krishnamurthy, P. C. Pattnaik, C.C. Tsuei, C. C. Chi, and C.L. Cane, Physica (Amsterdam) 186-188B, 801 (1993).

[6] D. A. Bonn et al., Phys. Rev. B 47. 11314 (1993).

17] F. Gao et al., Appl. Phys. Lett. 63, 2274 (1993).

[8] M. C. Nuss et al., Phys. Rev. Lett., 66, 3305 (1991).

[9] R. C. Yu et al., Phys. Rev. Lett. 69, 1431 (1992).

[10] C. D. Marshall et al.. J. Appl. Phys. 73, 850 (1993).

[11] C. D. Marshall et al., Phys. Rev. B. 43, 2696 (1991).

[12] A. I. Bezuglyi and V. A. Shklovskij (to be published).

[13] V. G. Volotskaya et al.. Sov. Phys. J. Low Temp. Phys. 18, 683 (1992).

[14] D. L. Novikov and A.J. Freeman, Physica (Amsterdam), 216C, 273 (1993).

[15] M. I. Flik et al., Phys. Rev. B 46, 5606 (1992).

[16] C. T. Rieck et al., Solid State Commun. 88, 395 (1993).

[17] E. J. Nicol and J.P. Carbotte Phys. Rev. B, 44, 7741 (1991).

[18] Nonequilibrium Superconductivity, Phonons, and Kapitza Boundaries edited by K.E. Gray (Plenum Press, New York. 1981).

[19] Nonequilibrium Superconductivity, edited by D.N. Langenberg and A. I. Larkin (North-Holland, Amsterdam. 1986). 